\title{
Automorphism Groups and Twisted Modules for Lattice Vertex Operator Algebras
}

\author{
Chongying Dong] \\ Department of Mathematics, University of California \\ Santa Cruz, CA 95064 \\ Kiyokazu Nagatomof \\ Department of Mathematics, Graduate School of Science \\ Osaka University, Toyonaka, Osaka 560-0048, Japan
}

\begin{abstract}
We give a complete description of the full automorphism group of a lattice vertex operator algebra, determine the twisted Zhu's algebra for the automorphism $\theta$ lifted from the -1 isometry of the lattice and classify the irreducible $\theta$-twisted modules through the twisted Zhu algebra.
\end{abstract}

\section{Introduction}

Vertex (operator) algebra $V_{L}$ associated with an arbitrary even lattice $L$ is one of the important motivations for introducing the notion of vertex (operator) algebra and also provides basic examples of such algebras ([B], [FLM]). The study of $V_{L}$ when $L$ is positive has been fruitful. If $L$ is positive definite $V_{L}$ is regular [DLM1] in the sense that any weak module is a direct sum of ordinary irreducible modules which have been classified (see [FLM] and [D1]). A characterization of $V_{L}$ was obtained in [LX]. The automorphism groups of all known vertex operator subalgebras of $V_{L}$ when the rank of $L$ is one are determined in [DG] and [DGR].

The vertex operator algebra $V_{L}$ has a canonical automorphism $\theta$ of order 2 lifted from the -1 isometry of $L$. The aim of this paper is to determine the full automorphism group Aut $\left(V_{L}\right)$ of $V_{L}$, compute the $\theta$-twisted Zhu's algebra [DLM3] and classify the irreducible $\theta$-twisted modules for $V_{L}$ by using the twisted Zhu's algebra when $L$ is positive definite.

\footnotetext{
${ }^{1}$ Supported by NSF grant DMS-9700923 and a research grant from the Committee on Research, UC Santa Cruz.

${ }^{2}$ Partly supported by Grant-in-Aid for Scientific Research, the Ministry of Education, Science and Culture.
} 
The group structure of Aut $\left(V_{L}\right)$ determined in this paper is important in understanding automorphism groups of vertex operator algebras in general. Let $V$ be a vertex operator algebra without vectors of negative weights and weight zero subspace being one dimensional. Then the weight one subspace $V_{1}$ is a Lie algebra under the bracket $[u, v]=u_{0} v$ where $u_{0}$ is the component operator of vertex operator $Y(u, z)=\sum_{n \in \mathbb{Z}} u_{n} z^{-n-1}$ associated to $u$. The exponentials $e^{u_{0}}$ for $u \in V_{1}$ generate a continuous, normal subgroup $N$ of Aut $(V)$. So the task is to determine the quotient group Aut $(V) / N$. In this paper we show that Aut $\left(V_{L}\right) / N$ is isomorphic to a quotient group of the finite group $O(L)$ which is the subgroup of Aut $(L)$ of isometries. This may suggest that Aut $(V) / N$ is a finite group in general. There are two extremes. Namely, $V_{1}$ generates $V$ in the sense of [FHL] or $V_{1}=0$. For example, the vertex operator algebras associated to affine Kac-Moody algebras (cf. [DL1], [FZ] and [Li]) are generated by weight one subspaces and $\operatorname{Aut}(V)$ is isomorphic to the automorphism group of the Lie algebra $V_{1}$; the moonshine vertex operator algebra $V^{\natural}[\mathrm{FLM}]$ and the vertex operator algebras associated to the Virasoro algebra (cf. [FZ] and [Li]) have zero weight one subspaces and the automorphism groups are the monster simple group and the identity.

The automorphisms studied in this paper preserve the Virasoro element. But it is also an important problem to investigate the automorphisms which need not preserve the Virasoro element. Such problem was first considered in $[\mathrm{MN}]$ in the case of free bosonic vertex algebra, where the the group of all such automorphisms of free bosonic vertex algebra was obtained. It is still an open problem to determine all such automorphisms for $V_{L}$.

In [DLM3], an associative algebra $A_{g}(V)$ was introduced for a vertex operator algebra $V$ and an automorphism $g$ of finite order so that there is a one to one correspondence between the equivalence classes of irreducible admissible $g$-twisted $V$-modules and the equivalence classes of irreducible $A_{g}(V)$-modules. In the case $g=1$ this amounts Zhu's original algebra $A(V)$ and related results. The Zhu's algebras $A(V)$ have been computed for the vertex operator algebras associated to affine Lie algebras in [FZ], the vertex operator algebras associated to the Virasoro algebras in [W] and for $V_{L}$ in [DLM2]. Our result on $A_{\theta}\left(V_{L}\right)$ in this paper is the first example of explicit calculation of twisted Zhu's algebra. It turns out the computation of $A_{\theta}\left(V_{L}\right)$ is much simpler than that of $A\left(V_{L}\right)$ and the structure of $A_{\theta}\left(V_{L}\right)$ is also simpler.

Let $\hat{L}$ be the canonical central extension of $L$ by a cyclic group $\langle \pm 1\rangle$ of order 2 . In 
[FLM] and [Le], a subgroup $K$ of $\hat{L}$, which is isomorphic to $2 L$, was introduced and all irreducible modules for $\hat{L} / K$ on which $(-1) K$ acts as -1 were classified and denoted by $T_{\chi}$. It was shown in [FLM] (also see [DL2]) each $T_{\chi}$ gives rise an irreducible $\theta$-twisted module. We show in this paper that $A_{\theta}\left(V_{L}\right)$ is isomorphic to a quotient of the group algebra $\mathbb{C}[\hat{L} / K]$ modulo the ideal generated by $(-1) K+1$. As a result we know all the irreducible modules of $A_{\theta}\left(V_{L}\right)$ are precisely these $T_{\chi}$. Using the theory of twisted Zhu's

algebra developed in [DLM3] we give a new proof of a result in [D2] on classification of irreducible $\theta$-twisted $V_{L}$-module. Although the proof in [D2] can be easily generalized to prove that $V_{L}$ is $\theta$-rational but this has not appeared in any publication. We also prove a general result which says that for an arbitrary vertex operator algebra $V$ and an automorphism $g$ of finite order such that $A_{g}(V)$ is finite-dimensional, semisimple and the weights of top levels of the all irreducible $g$-twisted $V$-modules are the same then $V$ is $g$-rational. As a corollary we show that $V_{L}$ is $\theta$-rational.

The most of this work was done when the second author was visiting UC Santa Cruz in academic year 1997-1998. The second author wishes to thank the Department of Mathematics here for their hospitality during his stay. The authors are grateful to Geoffrey Mason and Michael Tuite for helpful comments related to this work.

\section{Automorphism groups}

In this section we determine the automorphism group of the vertex operator algebras $V_{L}$ associated to even positive definite lattices $L$. In Subsection 2.1 we review the canonical central extension $\hat{L}$ of $L$ by the cyclic group of order 2 and the structure of the automorphism group of $\hat{L}$ following [FLM]. We recall the explicit construction of the vertex operator algebra $V_{L}$ from $[\mathrm{FLM}]$ in Subsection 2.2. Subsection 2.3 is on how to lift a certain derivations of a vertex operator algebra to automorphisms. Subsection 2.4 is the core of Section 2, where the automorphism group of $V_{L}$ is obtained by using the automorphism group of $\hat{L}$ and the automorphisms which are the exponentials of the weight zero components of the weight one vectors. 


\subsection{Integral lattices and their central extensions}

Let $L$ be any positive-definite even lattice and $\hat{L}$ be the canonical central extension of $L$ by the cyclic group $\langle \pm 1\rangle$ :

$$
1 \rightarrow\langle \pm 1\rangle \rightarrow \hat{L} \stackrel{-}{\rightarrow} \rightarrow 1
$$

with the commutator map $c(\alpha, \beta)=(-1)^{\langle\alpha, \beta\rangle}$ for $\alpha, \beta \in L$. Let $e: L \rightarrow \hat{L}$ be a section such that $e_{0}=1$ and $\epsilon: L \times L \rightarrow\langle \pm 1\rangle$ be the corresponding 2-cocycle. Then $\epsilon(\alpha, \beta) \epsilon(\beta, \alpha)=(-1)^{\langle\alpha, \beta\rangle}$,

$$
\epsilon(\alpha, \beta) \epsilon(\alpha+\beta, \gamma)=\epsilon(\beta, \gamma) \epsilon(\alpha, \beta+\gamma)
$$

and $e_{\alpha} e_{\beta}=\epsilon(\alpha, \beta) e_{\alpha+\beta}$ for $\alpha, \beta, \gamma \in L$. We will use Aut $(G)$ to denote the automorphism group of any group $G$. Following $[\mathrm{FLM}]$ we define

$$
\operatorname{Aut}(c)=\{\sigma \in \operatorname{Aut}(L) \mid c(\sigma \alpha, \sigma \beta)=c(\alpha, \beta) \text { for } \alpha, \beta \in L\} \text {. }
$$

Then we have the lifting property (cf. Proposition 5.4.1 of [FLM])

$$
1 \rightarrow \operatorname{Hom}(L, \mathbb{Z} / 2 \mathbb{Z}) \rightarrow \operatorname{Aut}(\widehat{L}) \stackrel{-}{\rightarrow} \operatorname{Aut}(c) \rightarrow 1 \quad(\text { exact }) .
$$

One can easily verify that

$$
\operatorname{Hom}(L, \mathbb{Z} / 2 \mathbb{Z}) \cong(\mathbb{Z} / 2 \mathbb{Z})^{\operatorname{rank} L} .
$$

\subsection{Lattice vertex operator algebras}

Let $\mathfrak{h}=\mathbb{C} \otimes_{\mathbb{Z}} L$ and extend the $\mathbb{Z}$-form on $L$ to $\mathfrak{h}$ by $\mathbb{C}$-linearity. The corresponding affine Lie algebra is $\hat{\mathfrak{h}}=\mathfrak{h} \otimes \mathbb{C}\left[t, t^{-1}\right] \oplus \mathbb{C} c$. We also use the notation $h(n)=h \otimes t^{n}$ for $h \in \mathfrak{h}, n \in \mathbb{Z}$. Set

$$
\hat{\mathfrak{h}}^{-}=\mathfrak{h} \otimes t^{-1} \mathbb{C}\left[t^{-1}\right]
$$

Then $\hat{\mathfrak{h}}^{-}$is an abelian subalgebra of $\hat{\mathfrak{h}}$. Let $U\left(\hat{\mathfrak{h}}^{-}\right)=S\left(\hat{\mathfrak{h}}^{-}\right)$be the universal enveloping algebra of $\hat{\mathfrak{h}}^{-}$. Consider the induced $\hat{\mathfrak{h}}$-module

$$
M(1)=U(\hat{\mathfrak{h}}) \otimes_{U(\mathbb{C}[t] \otimes \mathfrak{h} \oplus \mathbb{C} c)} \mathbb{C} \simeq S\left(\hat{\mathfrak{h}}^{-}\right) \text {(linearly), }
$$

where $\mathbb{C}[t] \otimes \mathfrak{h}$ acts trivially on $\mathbb{C}$ and $c$ acts on $\mathbb{C}$ as multiplication by 1 . 
Recall from (2.1) that $\hat{L}$ is the canonical central extension of $L$ by the cyclic group $\langle \pm 1\rangle$. Form the induced $\hat{L}$-module

$$
\mathbb{C}\{L\}=\mathbb{C}[\hat{L}] \otimes_{\langle \pm 1\rangle} \mathbb{C} \simeq \mathbb{C}[L] \text { (linearly) }
$$

where $\mathbb{C}[\cdot]$ denotes the group algebra and -1 acts on $\mathbf{C}$ as multiplication by -1 . For $a \in \hat{L}$, write $\iota(a)$ for $a \otimes 1$ in $\mathbb{C}\{L\}$. Then the action of $\hat{L}$ on $\mathbb{C}\{L\}$ is given by: $a \cdot \iota(b)=\iota(a b)$ and $(-1) \cdot \iota(b)=-\iota(b)$ for $a, b \in \hat{L}$.

Furthermore we define an action of $\mathfrak{h}$ on $\mathbb{C}\{L\}$ by: $h \cdot \iota(a)=\langle h, \bar{a}\rangle \iota(a)$ for $h \in \mathfrak{h}, a \in$ $\hat{L}$. Define $z^{h} \cdot \iota(a)=z^{\langle h, \bar{a}\rangle} \iota(a)$.

The untwisted space associated with $L$ is defined to be

$$
V_{L}=M(1) \otimes_{\mathbb{C}} \mathbb{C}\{L\}
$$

and the vertex algebra algebra structure on $V_{L}$ (see $[\mathrm{B}]$ and $[\mathrm{FLM}]$ ) is determined by

$$
\begin{gathered}
Y(h(-1) \mathbf{1}, z)=h(z)=\sum_{n \in \mathbb{Z}} h(n) z^{-n-1} \quad(h \in \mathfrak{h}) \\
Y(\iota(a), z)=\exp \left(-\sum_{n<0} \frac{\bar{a}(n)}{n} z^{-n}\right) \exp \left(-\sum_{n>0} \frac{\bar{a}(n)}{n} z^{-n}\right) a z^{\bar{a}} .
\end{gathered}
$$

The Virasoro element is given by

$$
\omega=\frac{1}{2} \sum_{i=1}^{d} h_{i}(-1)^{2} \mathbf{1}
$$

where $\left\{h_{i} \mid i=1, \ldots, d\right\}$ is an orthogonal basis of $\mathfrak{h}$. Expand $L(z)=Y(\omega, z)$

$$
L(z)=\sum_{n \in \mathbb{Z}} L(n) z^{-n-2}
$$

Then the component operator $L(0)$ defines the weight gradation on $V_{L}$ :

$$
V_{L}=\oplus_{n \in \mathbb{Z}}\left(V_{L}\right)_{n}, \quad\left(V_{L}\right)_{n}=\left\{v \in V_{L} \mid L(0) v=n v\right\}
$$

Note that $\operatorname{dim}\left(V_{L}\right)_{n}<\infty$ for each $n$. For example,

$$
\mathrm{wt}(h(-n) \mathbf{1})=n, \quad \text { wt }(\iota(a))=\frac{1}{2}\langle\bar{a}, \bar{a}\rangle .
$$

We should point out that $M(1)$ is a vertex operator subalgebra of $V_{L}$. 


\subsection{Derivations and automorphisms}

Let $V$ be a vertex operator algebra and $\omega$ the Virasoro element. An automorphism of $V$ is an isomorphism $\sigma: V \longrightarrow V$ of vector spaces which preserves all the products and the Virasoro element:

$$
\sigma\left(a_{n} b\right)=\sigma(a)_{n} \sigma(b), \quad \sigma(\omega)=\omega .
$$

Note that an automorphism of $V$ preserves each homogeneous subspace $V_{n}$ of $V$. The group of all automorphisms of the vertex operator algebra $V$ is denoted by Aut $(V)$.

A derivation of $V$ is an endomorphism $D: V \longrightarrow V$ such that

$$
D\left(a_{n} b\right)=D(a)_{n} b+a_{n} D(b) \text { for all } a, b \in V, n \in \mathbb{Z}
$$

and $D(\omega)=0$. In particular, $D$ preserves the gradation of $V$. So the exponential $e^{D}$ converges on $V$ and is well-defined. It is easy to see that $e^{D}$ is an automorphism.

Let $a \in V$ and wt $(a)=1$. Then $D=a_{0}$ is a derivation of $V$ and $e^{a_{0}}$ is an automorphism of $V$. Set

$$
N=\left\langle e^{a_{0}} \mid a \in V_{1}\right\rangle .
$$

Since $\sigma e^{a_{0}} \sigma^{-1}=e^{(\sigma a)_{0}}$ and wt $(\sigma(a))=1$ for any $\sigma \in \operatorname{Aut}(V), N$ is a normal subgroup of $\operatorname{Aut}(V)$.

\subsection{The automorphism group of $V_{L}$}

We first describe certain automorphisms of $V_{L}$. Set

$$
O(L)=\{\sigma \in \operatorname{Aut}(L) \mid\langle\sigma \alpha, \sigma \beta\rangle=\langle\alpha, \beta\rangle \text { for } \alpha, \beta \in L\}
$$

which is the subgroup of Aut $(L)$ of isometries. Define

$$
O(L)=\{\sigma \in \operatorname{Aut}(\hat{L}) \mid \bar{\sigma} \in O(L)\} .
$$

Then $O(\hat{L})$ is a subgroup of $\operatorname{Aut}(\hat{L})$ and $\operatorname{Hom}(L, \mathbb{Z} / 2 \mathbb{Z}$ ) is a subgroup of $O(\hat{L})$ (see (2.2)). It follows from (2.2) that we have an exact sequence

$$
1 \rightarrow \operatorname{Hom}(L, \mathbb{Z} / 2 \mathbb{Z}) \rightarrow O(\hat{L}) \stackrel{-}{\rightarrow} O(L) \rightarrow 1 .
$$

Every $\sigma \in O(\hat{L})$ induces an automorphism of $V_{L}$, denoting by $\sigma$ also, in the following way

$$
\sigma\left(\alpha_{1}\left(-n_{1}\right) \cdots \alpha_{k}\left(-n_{k}\right) \otimes \iota(a)\right)=\left(\bar{\sigma} \alpha_{1}\right)\left(-n_{1}\right) \cdots\left(\bar{\sigma} \alpha_{k}\right)\left(-n_{k}\right) \otimes \iota(\sigma a)
$$


for $\alpha_{i} \in \mathfrak{h}, n_{i}>0$ and $a \in \hat{L}$. So we can regard $O(\hat{L})$ as a subgroup of Aut $\left(V_{L}\right)$. Note that such automorphisms preserve the vertex operator subalgebra $M(1)$.

The first main result is the determination of the automorphism group of $V_{L}$.

Theorem 2.1. Let $L$ be a positive definite even lattice. Then

$$
\operatorname{Aut}\left(V_{L}\right)=N \cdot O(\hat{L})
$$

and $N$ is a normal subgroup of Aut $\left(V_{L}\right)$. Moreover, the intersection $N \cap O(\hat{L})$ contains a subgroup $\operatorname{Hom}(L, \mathbb{Z} / 2 \mathbb{Z})$ and $\operatorname{Aut}\left(V_{L}\right) / N$ is isomorphic to a quotient group of $O(L)$.

To prove this theorem, we need the following elementary lemmas.

Lemma 2.2. Let $0 \neq v \in V_{L}$. Suppose that there exists $\alpha \in \mathfrak{h}(\alpha \neq 0)$ such that $h(0) v=\langle h, \alpha\rangle v$ for all $h \in \mathfrak{h}$. Then $\alpha \in L$ and there exists $c_{\alpha} \in M(1)$ such that $v=c_{\alpha} \iota\left(e_{\alpha}\right)$.

Proof. Note that $\mathfrak{h}$ acts on $V_{L}$ semisimply,

$$
V_{L}=\bigoplus_{\beta \in L} M(1) \otimes \iota\left(e_{\beta}\right)
$$

and $M(1) \otimes \iota\left(e_{\beta}\right)$ is precisely the subspace with the eigenfunction $\beta$, that is

$$
M(1) \otimes \iota\left(e_{\beta}\right)=\left\{v \in V_{L} \mid h(0) v=\langle h, \beta\rangle v\right\} .
$$

The lemma follows immediately.

Set $\mathfrak{h}(-1)=\mathfrak{h} \otimes t^{-1} \mathbf{1}$, a subspace of $V_{L}$. Then any automorphism $\sigma$ of $V_{L}$ preserving $\mathfrak{h}(-1)$ can be considered as a linear automorphism of $\mathfrak{h}$.

Lemma 2.3. Let $\sigma \in \operatorname{Aut}\left(V_{L}\right)$ and suppose $\sigma(\mathfrak{h}(-1))=\mathfrak{h}(-1)$. Then $\sigma(L) \subset L$ and for any $\alpha \in L$, there exists $c_{\alpha} \in \mathbb{C}$ such that $\sigma\left(\iota\left(e_{\alpha}\right)\right)=c_{\alpha} \iota\left(e_{\sigma(\alpha)}\right)$.

Proof. From the assumption $\sigma^{-1}(h(-1) \mathbf{1}) \in \mathfrak{h}(-1)$ for any $h \in \mathfrak{h}$. Since the bilinear form on $\mathfrak{h}$ can be realized as $\langle u, v\rangle=u(1) v$ for $u, v \in \mathfrak{h}$ we see that the form $\langle$,$\rangle on \mathfrak{h}$ is $\sigma$-invariant. Here we have identified $\mathfrak{h}$ with $\mathfrak{h}(-1)$. Then we have from the definition of the action that $\sigma^{-1}(h)(0) \iota\left(e_{\alpha}\right)=\left\langle\alpha, \sigma^{-1}(h)\right\rangle \iota\left(e_{\alpha}\right)$. Thus

$$
\begin{aligned}
h(0) \sigma \iota\left(e_{\alpha}\right) & =\left(\sigma \sigma^{-1}(h)\right)(0) \sigma \iota\left(e_{\alpha}\right) \\
& =\sigma\left(\sigma^{-1}(h)(0) \iota\left(e_{\alpha}\right)\right) \\
& =\sigma\left(\left\langle\alpha, \sigma^{-1}(h)\right\rangle \iota\left(e_{\alpha}\right)\right) \\
& =\left\langle\alpha, \sigma^{-1}(h)\right\rangle \sigma \iota\left(e_{\alpha}\right) .
\end{aligned}
$$


This implies that $h(0) \sigma \iota\left(e_{\alpha}\right)=\langle h, \sigma(\alpha)\rangle \sigma \iota\left(e_{\alpha}\right)$. Clearly $\sigma\left(\iota\left(e_{\alpha}\right)\right) \neq 0$. Using Lemma 2.2 we see that there exists $c_{\alpha} \in M(1)$ such that $\sigma\left(\iota\left(e_{\alpha}\right)\right)=c_{\alpha} \iota\left(e_{\sigma(\alpha)}\right)$. In particular, $\sigma(L) \subset$ $L$. Note that $\mathbb{C}\{L\}$ is the vacuum space for the Heisenberg algebra $\oplus_{n \neq 0} t^{n} \otimes \mathfrak{h} \oplus \mathbb{C} c$ in the sense that

$$
\mathbb{C}\{L\}=\left\{v \in V_{L} \mid h(n) v=0, h \in \mathfrak{h}\right\} .
$$

So $\mathbb{C}\{L\}$ is $\sigma$-stable and $c_{\alpha} \in \mathbb{C}$.

Remark 2.4. Since the vectors $\iota\left(e_{\alpha}\right)$ and $\iota\left(e_{\sigma \alpha}\right)$ in the proof of Lemma 2.3 have the same weight, $\sigma$ induces an isometry of $L$.

Lemma 2.5. Let $\sigma \in \operatorname{Aut}\left(V_{L}\right)$ such that $\left.\sigma\right|_{\mathfrak{h}(-1)}=\mathrm{id}$. Then there exists $h \in \mathfrak{h}$ such that $\sigma=e^{h(0)}$.

Proof. Since $M(1)$ is generated by $\mathfrak{h}(-1)$ we see immediately that the restriction of $\sigma$ to $M(1)$ is the identity. Note that $\sigma$ is also the identity on $L$. By Lemma 2.3 for any $\alpha \in L$ there exists $c_{\alpha} \in \mathbb{C}$ so that $\sigma \iota\left(e_{\alpha}\right)=c_{\alpha} \iota\left(e_{\alpha}\right)$. Recall the component vertex operators $v_{n}$ of $Y(v, z)=\sum_{n \in \mathbb{Z}} v_{n} z^{-n-1}$ for any $v \in V_{L}$. Then for any $\alpha, \beta \in L$, $\iota\left(e_{\alpha}\right)_{\langle\alpha, \beta\rangle-1} \iota\left(e_{\beta}\right)=\epsilon(\alpha, \beta) \iota\left(e_{\alpha+\beta}\right)$. We see

$$
\begin{aligned}
\sigma \iota\left(e_{\alpha+\beta}\right) & =\epsilon(\alpha, \beta)^{-1} \sigma \iota\left(e_{\alpha}\right)_{\langle\alpha, \beta\rangle-1} \sigma \iota\left(e_{\beta}\right) \\
& =\epsilon(\alpha, \beta)^{-1} c_{\alpha} c_{\beta} \iota\left(e_{\alpha}\right)_{\langle\alpha, \beta\rangle-1} \iota\left(e_{\beta}\right) \\
& =c_{\alpha} c_{\beta} \iota\left(e_{\alpha+\beta}\right) .
\end{aligned}
$$

This shows that $c_{\alpha+\beta}=c_{\alpha} c_{\beta}$.

Let $\left\{\alpha_{i}\right\}_{i=1}^{d}$ be a basis of $L$. Then for $\alpha=\sum_{i=1}^{d} k_{i} \alpha_{i}\left(k_{i} \in \mathbb{Z}\right)$, we see $c_{\alpha}=$ $c_{1}^{k_{1}} c_{2}^{k_{2}} \cdots c_{d}^{k_{d}}$. Since the inner product is non-degenerate, there exists $h \in \mathfrak{h}$ such that $e^{\left\langle h, \alpha_{i}\right\rangle}=c_{\alpha_{i}}$ for all $i=1,2, \ldots, d$. Then $e^{h(0)} \iota\left(e_{\alpha_{i}}\right)=c_{\alpha_{i}} \iota\left(e_{\alpha_{i}}\right)$ for all $i=1,2, \ldots, d$ and

$$
e^{h(0)} \iota\left(e_{\alpha}\right)=c_{\alpha_{1}}^{k_{1}} \cdots c_{\alpha_{d}}^{k_{d}} \iota\left(e_{\alpha}\right)=c_{\alpha} \iota\left(e_{\alpha}\right) .
$$

Since both $\sigma$ and $e^{h(0)}$ act trivially on $\mathfrak{h}(-1)$ and have the same action on $\mathbb{C}\{L\}$, we conclude that $\sigma=e^{h(0)}$.

Proof of Theorem 2.1. Let us consider the weight one space

$$
\mathfrak{g}=V_{L}(1)=\mathfrak{h} \oplus \sum_{\langle\alpha, \alpha\rangle=2} \mathbb{C} \iota\left(e_{\alpha}\right)
$$


which is a finite dimensional reductive Lie algebra under the bracket $[u, v]=u_{0} v$ for $u, v \in \mathfrak{g}$ with a Cartan subalgebra $\mathfrak{h}=\mathfrak{h}(-1)$.

Let $\sigma$ be an automorphism of $V_{L}$. Then $\sigma$ induces an automorphism of the Lie algebra $\mathfrak{g}$. Note that the inner automorphism group of $\mathfrak{g}$ is the restriction of $N$ to $\mathfrak{g}$. The conjugacy theorem for Cartan subalgebras of finite dimensional reductive Lie algebras implies that there exists $\phi \in N$ such that $\phi^{-1} \sigma(\mathfrak{h})=\mathfrak{h}$. Then by Lemma 2.3 and Remark 2.4, $\mu=\phi^{-1} \sigma$ induces an isometry of $L$ and $\mu \iota\left(e_{\alpha}\right)=c_{\alpha} \iota\left(e_{\mu \alpha}\right)$ for $\alpha \in L$ and some constant $c_{\alpha} \in \mathbb{C}$. Let $\tau \in O(\hat{L})$ such that $\bar{\tau}=\mu$. Then $\mu \tau^{-1}$ is the identity when restricted to $\mathfrak{h}(-1)$. By Lemma 2.3 there exists $\psi \in N$ such that $\mu \tau^{-1}=\psi$. That is, $\sigma=\phi \psi \tau \in N \cdot O(\widehat{L})$ as $\phi \psi \in N$. This shows that $\operatorname{Aut}\left(V_{L}\right)=N \cdot O(\hat{L})$.

In order to see $\operatorname{Hom}(L, \mathbb{Z} / 2 \mathbb{Z})$ is a subgroup of $N$ we recall from [FLM] on how $\operatorname{Hom}(L, \mathbb{Z} / 2 \mathbb{Z})$ is embedded into $\operatorname{Aut}(\hat{L})$. Let $\lambda \in \operatorname{Hom}(L, \mathbb{Z} / 2 \mathbb{Z})$. The corresponding automorphism of $\hat{L}$ is given by: $a \mapsto a(-1)^{\lambda(\bar{a})}$ for $a \in \hat{L}$. As in the proof of Lemma 2.5 there exists $h \in \mathfrak{h}$ such that $e^{\langle h, \alpha\rangle}=(-1)^{\lambda(\alpha)}$ for $\alpha \in L$. It is easy to see that the $\lambda$ and $e^{h(0)}$ give the same automorphism of $V_{L}$ and thus $\lambda \in N$.

An isomorphism from Aut $\left(V_{L}\right) / N$ to a quotient group of $O(L)$ follows from the facts that $\operatorname{Aut}\left(V_{L}\right) / N$ is isomorphic to $O(\hat{L}) / N \cap O(\hat{L})$, that $N \cap O(\hat{L})$ contains $\operatorname{Hom}(L, \mathbb{Z} / 2 \mathbb{Z})$ and that $O(\hat{L}) / \operatorname{Hom}(L, \mathbb{Z} / 2 \mathbb{Z})$ is isomorphic to $O(L)$.

\section{$3 \quad \theta$-twisted modules}

Let $V_{L}$ be as before. Fix an involution $\theta$ of $\hat{L}$ of order 2 such that $\bar{\theta}=-1$ on $L$. For example, one can define $\theta(a)=a^{-1}(-1)^{\langle\bar{a}, \bar{a}\rangle / 2}$ for $a \in \hat{L}$. Then $\theta$ is an automorphism of $V_{L}$ and $M(1)$. We determine twisted Zhu's algebra $A_{\theta}\left(V_{L}\right)$ and $A_{\theta}(M(1))$ explicitly in this section. We also classify the irreducible $\theta$-twisted modules for these vertex operator algebras by using $A_{\theta}\left(V_{L}\right)$ and $A_{\theta}(M(1))$ and prove the $\theta$-rationality of $V_{L}$.

Beginning with a arbitrary vertex operator algebra $V$ and an automorphism $g$ of finite order $g$ in Subsection 3.1, we recall the definitions of admissible $g$-twisted modules for $V$ and twisted Zhu's algebra $A_{g}(V)$ and related results from [DLM3]. The $g$-rationality of $V$ is established under certain assumption on the structure of $A_{g}(V)$. In Subsection 3.2 we give the construction of $\theta$-twisted modules for $V_{L}$ following [FLM]. In Subsection 3.3 we obtain a finite spanning set of $A_{\theta}\left(V_{L}\right)$ indexed by $L / 2 L$ and prove that $A_{\theta}(M(1))$ is isomorphic to the one-dimensional associative algebra $\mathbb{C}$. We show in Subsection 3.4 that $A_{\theta}\left(V_{L}\right)$ is isomorphic to a quotient of the group algebra $\mathbb{C}[\hat{L} / K]$ 
where $K$ is a subgroup of $\hat{L}$ (defined in Subsection 3.2) isomorphic to $2 L$. Using this result we prove that every irreducible $\theta$-twisted $V_{L}$-module is isomorphic to $V_{L}^{T_{\chi}}$ for some $\hat{L} / K$-modules $T_{\chi}$ (see Subsection 3.3 for the definition of $T_{\chi}$ ). The $\theta$-rationality of $V_{L}$ is an easy corollary of a general result on $g$-rationality in Subsection 3.1.

\subsection{Twisted modules and twisted Zhu algebras}

Let $V$ be a vertex operator algebra and $g$ an automorphism of $V$ of finite order $T$. Decompose $V$ into $g$-eigenspaces: $V=\bigoplus_{r \in \mathbb{Z} / T \mathbb{Z}} V^{r}$ where $V^{r}=\left\{v \in V \mid g v=e^{-2 \pi i r / T} v\right\}$.

An admissible $g$-twisted $V$-module (cf. [DLM3], [Z]) is a $\frac{1}{T} \mathbb{Z}$-graded vector space

$$
M=\sum_{n=0}^{\infty} M\left(\frac{n}{T}\right)
$$

with top level $M(0) \neq 0$, equipped with a linear map

$$
\begin{aligned}
& V \rightarrow(\text { End } M)\{z\} \\
& v \mapsto Y_{M}(v, z)=\sum_{n \in \mathbb{Q}} v_{n} z^{-n-1} \quad\left(v_{n} \in \text { End } M\right)
\end{aligned}
$$

satisfying the following conditions; for all $0 \leq r \leq T-1, u \in V^{r}, v \in V, w \in M$,

$$
\begin{gathered}
Y_{M}(u, z)=\sum_{n \in \frac{r}{T}+\mathbb{Z}} u_{n} z^{-n-1}, \\
u_{n} w=0 \text { for } n \gg 0, \\
Y_{M}(\mathbf{1}, z)=1, \\
z_{0}^{-1} \delta\left(\frac{z_{1}-z_{2}}{z_{0}}\right) Y_{M}\left(u, z_{1}\right) Y_{M}\left(v, z_{2}\right)-z_{0}^{-1} \delta\left(\frac{z_{2}-z_{1}}{-z_{0}}\right) Y_{M}\left(v, z_{2}\right) Y_{M}\left(u, z_{1}\right) \\
=z_{2}^{-1}\left(\frac{z_{1}-z_{0}}{z_{2}}\right)^{-r / T} \delta\left(\frac{z_{1}-z_{0}}{z_{2}}\right) Y_{M}\left(Y\left(u, z_{0}\right) v, z_{2}\right),
\end{gathered}
$$

where $\delta(z)=\sum_{n \in \mathbb{Z}} z^{n}$ and all binomial expressions are to be expanded in nonnegative integral powers of the second variable;

$$
u_{m} M(n) \subset M(\text { wt }(u)-m-1+n)
$$

if $u$ is homogeneous.

A $g$-twisted $V$-module is an admissible $g$-twisted $V$-module $M$ which carries a $\mathbb{C}$ grading by weight. That is, we have

$$
M=\coprod_{\lambda \in \mathbb{C}} M_{\lambda}
$$


where $M_{\lambda}=\{w \in M \mid L(0) w=\lambda w\}$. Moreover we require that $\operatorname{dim} M_{\lambda}$ is finite and for fixed $\lambda, M_{\lambda+\frac{n}{T}}=0$ for all small enough integers $n$.

$V$ is called $g$-rational if any admissible $g$-twisted module for $V$ is a direct sum of irreducible $g$-twisted $V$-modules.

Nest we review twisted Zhu's algebra [DLM3]. For homogeneous $u \in V^{r}$ and $v \in V$ we define

$$
u \circ_{g} v=\operatorname{Res}_{z} \frac{(1+z)^{\mathrm{wt}(u)-1+\delta_{r}+\frac{r}{T}}}{z^{1+\delta_{r}}} Y(u, z) v
$$

where $\delta_{r}=1$ if $r=0$ and $\delta_{r}=0$ if $r \neq 0$ and where, here and below, $(1+z)^{\alpha}$ for $\alpha \in \mathbb{C}$ is to be expanded in nonnegative integer powers of $z$. Let $O_{g}(V)$ be the linear span of all $u \circ_{g} v$ and define the linear space $A_{g}(V)$ to be the quotient $V / O_{g}(V)$.

Lemma 3.1. (1) Assume that $u \in V^{r}$ is homogeneous, $v \in V$ and $m \geq n \geq 0$. Then

$$
\operatorname{Res}_{z} \frac{(1+z)^{\mathrm{wt}(u)-1+\delta_{r}+\frac{r}{T}+n}}{z^{m+\delta_{r}+1}} Y(u, z) v \in O_{g}(V) .
$$

(2) $V^{r}$ is a subspace of $O_{g}(V)$ if $r \neq 0$.

Let us define the second product $*_{g}$ on $V$. For $r, u$ and $v$ as above, set

$$
u *_{g} v= \begin{cases}\operatorname{Res}_{z} Y(u, z) \frac{(1+z)^{\mathrm{wt}(u)}}{z} v & \text { if } r=0 \\ 0 & \text { if } r>0\end{cases}
$$

Extend this to a bilinear product on $V$.

Let $M$ is an admissible $g$-twisted $V$-module. Define the "vacuum space" $\Omega(M)$ of $M$ to be

$$
\left\{w \in M \mid u_{n} w=0, n>\operatorname{wt}(u)-1, n \in \frac{1}{T} \mathbb{Z}\right\} .
$$

Then $M(0) \subset \Omega(M)$ and the weight zero operator $o(v)$, which is equal to $v_{\mathrm{wt}}(v)-1$ when $v$ is homogeneous, acts on $\Omega(M)$.

Theorem 3.2. [DLM3] (1) The product $*_{g}$ induces the structure of an associative algebra on $A_{g}(V)$.

(2) For any admissible g-twisted $V$-module $M, v+O_{g}(V) \mapsto \operatorname{End} \Omega(M)$ gives an $A_{g}(V)$-module structure on $\Omega(M)$ and $M(0)$ is a submodule of $\Omega(M)$.

(3) $M \mapsto M(0)$ gives a bijection between the equivalence classes of irreducible $g$ twisted admissible $V$-modules and the equivalence classes of simple $A g(V)$-modules.

(4) If $V$ is g-rational, $A_{g}(V)$ is a finite-dimensional semisimple algebra. 
The following theorem is new.

Theorem 3.3. Let $V$ be a vertex operator algebra and $g$ an automorphism of order $T$ such that $A_{g}(V)$ is a finite-dimensional semisimple algebra and $\omega+O_{g}(V)$ acts on the all irreducible modules as the same constant $\lambda$. Then $V$ is g-rational.

Proof. By definition of $g$-rationality we need to prove that any admissible $g$-twisted $V$-module $M$ is completely reducible.

First we show that if $M(0)$ is an irreducible $A_{g}(V)$-module and $M$ is generated by $M(0)$ then $M$ is irreducible. In this case $M=\bigoplus_{n \in \frac{1}{T} \mathbb{Z}, n \geq 0} M_{\lambda+n}$. If $M$ is not irreducible it contains a submodule $W=\oplus_{n \geq n_{0}>0} W_{\lambda+n}$ for some $n_{0}>0$ as $M(0)$ is an irreducible $A_{g}(V)$-module where $W_{\mu}=M_{\mu} \cap W$ and $W_{\lambda+n_{0}} \neq 0$. Then $W_{\lambda+n_{0}}$ is a module for $A_{g}(V)$ on which $\omega+O_{g}(V)$ acts as $\lambda+n_{0} \neq \lambda$. Since $A_{g}(V)$ is a finite-dimensional semisimple algebra, there exists an irreducible submodule of $W_{\lambda+n_{0}}$ on which $\omega+O_{g}(V)$ acts as $\lambda+n_{0} \neq \lambda$. This is a contradiction.

Now we consider general $M$. Let $X$ be the submodule of $M$ generated by $\Omega(M)$. Since $\Omega(M)$ is a direct sum of irreducible $A_{g}(V)$-module, $X$ is completely reducible by the argument above. Note the $X$ is the maximal completely reducible submodule of $M$. We claim that $X=M$.

If $\bar{M}=M / X \neq 0$. Then $\Omega(\bar{M}) \neq 0$. We can assume that $\bar{M}$ is completely reducible (otherwise replace $\bar{M}$ by the submodule generated by $\Omega(\bar{M})$ ). Thus any vector of $M$ is contained in a finite-dimensional $L(0)$-stable subspace and $M$ is a direct sum of generalized eigenspaces for $L(0)$ :

$$
M=\oplus_{n \in \frac{1}{T} \mathbb{Z}, n \geq 0} M_{\lambda+n}
$$

where $M_{\mu}$ is the generalized eigenspace with the eigenvalue $\mu$. Since $M_{\lambda}$ is a completely reducible $A_{g}(V)$-module, the submodule $W$ generated by $M_{\lambda}$ is completely reducible. If $M \neq W$ then $\Omega(M / W)$ contains a $A_{g}(V)$-submodule on which $\omega+O_{g}(V)$ acts as a constant which is greater than $\lambda$. Thus $M$ is completely reducible and $M=X$. This is a contradiction.

\subsection{The $\theta$-twisted modules}

We return to $V_{L}$. Recall that $\theta$ is an automorphism of $\hat{L}$ and $V_{L}$ such that $\theta(a)=$ $a^{-1}(-1)^{\langle\bar{a}, \bar{a}\rangle / 2}$ for $a \in \hat{L}$. Define $K=\left\{\theta(a) a^{-1} \mid a \in \widehat{L}\right\}$. Then $\bar{K}=2 L$. Also set 
$R=\{\alpha \in L \mid\langle\alpha, L\rangle \subset 2 \mathbb{Z}\} \supset 2 L$. Then the pull back $\widehat{R}$ of $R$ in $\widehat{L}$ is in the center of $\widehat{L}$ and $K$ is a subgroup of $\widehat{R}$.

The following proposition can be found in [FLM] (Proposition 7.4.8).

Proposition 3.4. There are exactly $|R / 2 L|$ central characters $\chi: \widehat{R} / K \longrightarrow \mathbb{C}^{\times}$of $\widehat{L} / K$ such that $\chi((-1) K)=-1$. For each such $\chi$, there is a unique (up to equivalence) irreducible $\widehat{L} / K$-module $T_{\chi}$ with central character $\chi$ and every irreducible $\widehat{L} / K$-module on which $(-1) K$ acts -1 is equivalent to ones of these. In particular, viewing $T_{\chi}$ as an $\widehat{L}$-module, $\theta(a)=a$ on $T_{\chi}$ for $a \in \widehat{L}$.

For each $T_{\chi}$, we define a twisted space

$$
V_{L}^{T_{\chi}}=M_{\mathbb{Z}+\frac{1}{2}}(1) \otimes T_{\chi} \cong S\left(\widehat{\mathfrak{h}}_{\mathbb{Z}+\frac{1}{2}}^{-}\right) \otimes T_{\chi}
$$

where $M_{\mathbb{Z}+\frac{1}{2}}(1)$ is the module for the Heisenberg algebra $\widehat{\mathfrak{h}}_{\mathbb{Z}+\frac{1}{2}}=\mathfrak{h} \otimes t^{1 / 2} \mathbb{C}\left[t, t^{-1}\right] \oplus \mathbb{C} c$ defined by

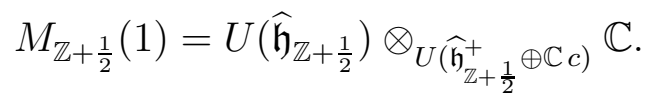

Here $\widehat{\mathfrak{h}}_{\mathbb{Z}+\frac{1}{2}}^{+}=\mathfrak{h} \otimes t^{1 / 2} \mathbb{C}[t]$ acts trivially on $\mathbb{C}$ and $c$ acts as 1 .

The $\theta$-twisted vertex operators $Y_{\theta}(\iota(a), z)$ for $a \in \hat{L}$ are defined to be

$$
Y_{\theta}(\iota(a), z)=2^{-\langle\bar{a}, \bar{a}\rangle} \circ e^{\int \bar{a}(z)} \circ a z^{-\langle\bar{a}, \bar{a}\rangle / 2}
$$

where $\alpha(z)=\sum_{n \in \mathbb{Z}+\frac{1}{2}} \alpha(n) z^{-n-1}$ and $\alpha(n)=\alpha \otimes t^{n}$ (see [FLM] for the detail). For $v=\alpha_{1}\left(-n_{1}\right) \cdots \alpha_{k}\left(-n_{k}\right) \otimes \iota(a) \in V_{L}$ homogeneous, set

$$
W_{\theta}(v, z)=\circ \partial^{\left(n_{1}-1\right)} \alpha_{1}(z) \cdots \partial^{\left(n_{k}-1\right)} \alpha_{k}(z) Y_{\theta}(\iota(a), z) \circ
$$

where $\partial^{(n)}=\frac{1}{n !}\left(\frac{d}{d z}\right)^{n}$. Now we extend this to all $v \in V_{L}$ by linearity. Recall that $\left\{h_{1}, \ldots, h_{d}\right\}$ be an orthonormal basis of $\mathfrak{h}$ and define constants $c_{m n} \in \mathbb{Z}$ for $m, n \geq 0$ by the formula

$$
\sum_{m, n \geq 0} c_{m n} x^{m} y^{n}=-\log \left(\frac{(1+x)^{\frac{1}{2}}+(1+y)^{\frac{1}{2}}}{2}\right) .
$$

Set

$$
\Delta_{z}=\sum_{m, n \geq 0} \sum_{i=1}^{d} c_{m n} h_{i}(m) h_{i}(n) z^{-m-n} .
$$

We finally define twisted vertex operator $Y_{\theta}(v, z)$ for $v \in V_{L}$ as

$$
Y_{\theta}(v, z)=W_{\theta}\left(e^{\Delta_{z}} v, z\right) .
$$

The following theorem was proved in [FLM] (see also [DL2]). 
Theorem 3.5. (1) For each $T_{\chi}$, the space $\left(Y_{\theta}, V_{L}^{T_{\chi}}\right)$ is an irreducible $\theta$-twisted module for the vertex operator algebra $V_{L}$.

(2) $M_{\mathbb{Z}+\frac{1}{2}}(1)$ is an irreducible $\theta$-twisted $M(1)$-module.

Let $W$ be an $\theta$-stable subspace of $V_{L}$. Decompose $W$ into eigenspaces with respect to the action of $\theta$ :

$$
W=W^{+} \oplus W^{-}
$$

where

$$
W^{ \pm}=\{v \in W \mid \theta(v)= \pm v\}
$$

Lemma 3.6. Let $W=M(1)$ or $V_{L}$. Suppose $u \in W^{-}$and $\mathrm{wt}(u)=1$. Then

$$
\sum_{j=0}^{\infty}\left(\begin{array}{c}
\frac{1}{2} \\
j
\end{array}\right) u_{j-m-1} v \in O_{\theta}(W) .
$$

Proof. Take $n=0$ in Lemma 3.1 (1), then we see

$$
\operatorname{Res}_{z} \frac{(1+z)^{\frac{1}{2}}}{z^{1+m}} Y(u, z) v \in O_{\theta}(W) .
$$

Substituting $(1+z)^{\frac{1}{2}}=\sum_{j=0}^{\infty}\left(\begin{array}{c}\frac{1}{2} \\ j\end{array}\right) z^{j}$ into the above, we get the result.

\subsection{A spanning set of $A_{\theta}\left(V_{L}\right)$}

Lemma 3.7. Let $W=M(1)$ or $V_{L}$. Let $\alpha \in \mathfrak{h}$ and $m \in \mathbb{Z}_{\geq 0}$. Then for any $v \in W$, there exist scalars $c_{m, j} \in \mathbb{C}$ for $j=0,1,2, \ldots$ such that

$$
\alpha(-m-1) v \equiv \sum_{j=0}^{\infty} c_{m, j} \alpha(j) v \quad \bmod O_{\theta}(W) .
$$

Proof. We prove the lemma by induction on $m$. Since $\alpha \in W^{-}$, setting $m=0$ in Lemma 3.6 gives

$$
\sum_{j=0}^{\infty}\left(\begin{array}{c}
\frac{1}{2} \\
j
\end{array}\right) \alpha(j-1) v \in O_{\theta}(W)
$$

Namely,

$$
\alpha(-1) v \equiv-\sum_{j=0}^{\infty}\left(\begin{array}{c}
\frac{1}{2} \\
j+1
\end{array}\right) \alpha(j) v \quad \bmod O_{\theta}(W) .
$$

Then $m=0$ case is done. 
Let $m>0$ and assume that the lemma is true for all nonnegative integers strictly less than $m$. By Lemma 3.6, we see

$$
\alpha(-m-1) v \equiv-\sum_{j=0}^{\infty}\left(\begin{array}{c}
\frac{1}{2} \\
j+1
\end{array}\right) \alpha(j-m) v \quad \bmod O_{\theta}(W) .
$$

Using the induction hypothesis completes the proof of the lemma.

Consider an element

$$
\alpha_{1}\left(-n_{1}\right) \cdots \alpha_{k}\left(-n_{k}\right) \otimes \iota(a)
$$

for $\alpha_{i} \in L, n_{1} \geq \cdots \geq n_{k}>0$ and $a \in \hat{L}$. We say the vector $v$ has the length $k$ and denote it by $\ell(v)$.

Lemma 3.8. Let $v \in M(1) \otimes \iota(a)$ for $a \in \hat{L}$. Then $v \equiv c_{v} \iota(a) \bmod O_{\theta}\left(V_{L}\right)$ for some scalar $c_{v} \in \mathbb{C}$. Moreover, if $a=1$ then $v \equiv c_{v} \bmod O_{\theta}(M(1))$.

Proof. We prove the lemma by induction on the length of $v$. Clearly it is enough to show the lemma for homogeneous elements. If $\ell(v)=0$, the lemma is obviously true. Suppose that the lemma is true for any homogeneous elements whose lengths are strictly less than $\ell(v)=k>0$. Then $v$ has the form $v=\alpha(-m-1) v^{\prime}$ for some $m \in \mathbb{Z}_{\geq 0}$ and $\alpha \in L$ where $v^{\prime} \in M(1) \otimes \iota(a)$ and $\ell\left(v^{\prime}\right)=k-1$. Then by Lemma 3.7, we see

$$
v \equiv \sum_{j=0}^{\infty} c_{m, j} \alpha(j) v^{\prime} \quad \bmod O_{\theta}\left(V_{L}\right)
$$

Since $\ell\left(\alpha(j) v^{\prime}\right)<t$ for all $j \geq 0$, by induction hypothesis, we prove the lemma.

If $a=1$ the result is clear now.

From Theorems 3.2, 3.5 and Lemma 3.8 we immediately have

Corollary 3.9. $A_{\theta}(M(1))$ is isomorphic to $\mathbb{C}$ and $M_{\mathbb{Z}+\frac{1}{2}}(1)$ is the unique irreducible $\theta$-twisted $M(1)$-module.

Recall that $e: L \rightarrow \hat{L}$ is a section. We may and do assume that $e_{2 \alpha} \in K$.

Lemma 3.10. Let $\alpha \in L$.

(1) We have $\iota\left(e_{2 \alpha}\right) \equiv 2^{-4\langle\alpha, \alpha\rangle} \mathbf{1}$ modulo $O_{\theta}\left(V_{L}\right)$.

(2) For any $v \in M(1) \otimes \iota\left(e_{-\alpha}\right), v \equiv c_{v} \iota\left(e_{\alpha}\right) \bmod O_{\theta}\left(V_{L}\right)$ for some scalar $c_{v} \in \mathbb{C}$. 
Proof. (1) Let $E^{\alpha}=\iota\left(e_{\alpha}\right)+\iota\left(\theta e_{\alpha}\right)$. Then clearly $E^{\alpha} \in V_{L}^{+}$and wt $\left(E^{\alpha}\right)=\langle\alpha, \alpha\rangle / 2$. Then by Lemma 3.1 (1), we see that for $m \in \mathbb{Z}_{\geq 0}$ and $v \in V_{L}$

$$
E_{-m-2}^{\alpha} v \equiv-\sum_{j=1}^{\frac{1}{2}\langle\alpha, \alpha\rangle}\left(\begin{array}{c}
\frac{1}{2}\langle\alpha, \alpha\rangle \\
j
\end{array}\right) E_{j-m-2}^{\alpha} v \quad \bmod O_{\theta}\left(V_{L}\right)
$$

Let $m=\langle\alpha, \alpha\rangle-1$ and $v=\iota\left(e_{\alpha}\right)$. Note that $\iota\left(e_{\alpha}\right)_{j-\langle\alpha, \alpha\rangle-1} \iota\left(e_{\alpha}\right)=\delta_{j 0} \epsilon(\alpha, \alpha) \iota\left(e_{2 \alpha}\right)$ for $j \geq 0$. Then we see

$$
\begin{aligned}
& \epsilon(\alpha, \alpha) \iota\left(e_{2 \alpha}\right)+\iota\left(\theta e_{\alpha}\right)_{-\langle\alpha, \alpha\rangle-1} \iota\left(e_{\alpha}\right) \\
& \quad \equiv-\sum_{j=1}^{\frac{1}{2}\langle\alpha, \alpha\rangle}\left(\begin{array}{c}
\frac{1}{2}\langle\alpha, \alpha\rangle \\
j
\end{array}\right) \iota\left(\theta e_{\alpha}\right)_{j-\langle\alpha, \alpha\rangle-1} \iota\left(e_{\alpha}\right) \quad \bmod O_{\theta}\left(V_{L}\right) .
\end{aligned}
$$

Since $\iota\left(\theta e_{\alpha}\right)_{j} \iota\left(e_{\alpha}\right) \in M(1)$ for all $j$ we see from Lemma 3.8 that $\iota\left(e_{2 \alpha}\right) \equiv c_{\alpha} \mathbf{1}$ for some scalar $c_{\alpha} \in \mathbb{C}$. Finally evaluation of this relation on top levels of all modules $V_{L}^{T_{\chi}}$ gives $c_{\alpha}=2^{-4\langle\alpha, \alpha\rangle}$.

For (2) we first use Lemma 3.8 to get a constant $d_{\alpha}$ such that $v \equiv d_{v} \iota\left(e_{-\alpha}\right)$ modulo $O_{\theta}\left(V_{L}\right)$. Note that $\iota\left(e_{-\alpha}\right)= \pm \iota\left(\theta e_{\alpha}\right)$. Since $\iota\left(e_{\alpha}\right)-\iota\left(\theta e_{\alpha}\right) \in V_{L}^{-} \subset O_{\theta}\left(V_{L}\right)$ we conclude $\iota\left(e_{-\alpha}\right) \equiv \pm \iota\left(e_{\alpha}\right)$ modulo $O_{\theta}\left(V_{L}\right)$, which finishes the proof of $(2)$.

Let $L=\cup_{i \in L / 2 L}\left(2 L+\beta_{i}\right)$ be the coset decomposition of $2 L$ in $L$. Then

Proposition 3.11. $A_{\theta}\left(V_{L}\right)$ is spanned by

$$
\left\{\iota\left(e_{\beta_{i}}\right)+O_{\theta}\left(V_{L}\right) \mid i \in L / 2 L\right\} .
$$

\subsection{Algebraic structure of $A_{\theta}\left(V_{L}\right)$}

Define the elementary Shur polynomials $p_{r}\left(x_{1}, x_{2}, \ldots\right), r \in \mathbb{Z}_{\geq 0}$ in variables $x_{1}, x_{2}, \ldots$ by the equation

$$
\exp \left(\sum_{n=1}^{\infty} \frac{x_{n}}{n} y^{n}\right)=\sum_{r=0}^{\infty} p_{r}\left(x_{1}, x_{2}, \ldots\right) y^{r} .
$$

Let $\alpha, \beta \in L$. Then

$$
\begin{aligned}
Y\left(\iota\left(e_{\alpha}\right), z\right) \iota\left(e_{\beta}\right) & =\epsilon(\alpha, \beta) z^{\langle\alpha, \beta\rangle} \exp \left(\sum_{n=1}^{\infty} \frac{\alpha(-n)}{n} z^{n}\right) \iota\left(e_{\alpha+\beta}\right) \\
& =\epsilon(\alpha, \beta) \sum_{r=0}^{\infty} p_{r}(\alpha(-1), \alpha(-2), \ldots) \iota\left(e_{\alpha+\beta}\right) z^{r+\langle\alpha, \beta\rangle} .
\end{aligned}
$$


Thus

$$
\begin{aligned}
& \iota\left(e_{\alpha}\right)_{i-1} \iota\left(e_{\beta}\right)=0 \quad(i \geq-\langle\alpha, \beta\rangle+1) \\
& \iota\left(e_{\alpha}\right)_{i-1} \iota\left(e_{\beta}\right)=\epsilon(\alpha, \beta) p_{-i-\langle\alpha, \beta\rangle}(\alpha(-1), \alpha(-2), \ldots) \iota\left(e_{\alpha+\beta}\right) \quad(i \leq-\langle\alpha, \beta\rangle) .
\end{aligned}
$$

By using these formulas, we will calculate $\iota\left(e_{\alpha}\right) *_{\theta} \iota\left(e_{\beta}\right)$ as follows. Set

$$
E^{\alpha}=\frac{1}{2}\left(\iota\left(e_{\alpha}\right)+\iota\left(\theta e_{\alpha}\right)\right), \quad F^{\alpha}=\frac{1}{2}\left(\iota\left(e_{\alpha}\right)-\iota\left(\theta e_{\alpha}\right)\right) .
$$

Then $E^{\alpha} \in V_{L}^{+}, F^{\alpha} \in V_{L}^{-}$and $\iota\left(e_{\alpha}\right)=E^{\alpha}+F^{\alpha}$. From the definition of the $*_{\theta}$ operation and Lemma 3.1, we have

$$
V_{L}^{+} *_{\theta} V_{L}^{+} \subset V_{L}^{+}, \quad V_{L}^{+} *_{\theta} V_{L}^{-} \subset V_{L}^{-} \subset O_{\theta}\left(V_{L}\right), \quad V_{L}^{-} *_{\theta} V_{L}=0
$$

Thus

$$
\iota\left(e_{\alpha}\right) *_{\theta} \iota\left(e_{\beta}\right) \equiv \operatorname{Res}_{z} \frac{(1+z)^{\frac{1}{2}\langle\alpha, \alpha\rangle}}{z} Y\left(E^{\alpha}, z\right) E^{\beta} \quad \bmod O_{\theta}\left(V_{L}\right) .
$$

More exactly, by using component operators,

$$
\iota\left(e_{\alpha}\right) *_{\theta} \iota\left(e_{\beta}\right) \equiv \sum_{i=0}^{\frac{1}{2}\langle\alpha, \alpha\rangle}\left(\begin{array}{c}
\frac{1}{2}\langle\alpha, \alpha\rangle \\
i
\end{array}\right) E_{i-1}^{\alpha} E^{\beta} \quad \bmod O_{\theta}\left(V_{L}\right)
$$

where

$$
\begin{aligned}
E_{i-1}^{\alpha} E^{\beta}= & \frac{1}{4}\left(\iota\left(e_{\alpha}\right)_{i-1} \iota\left(e_{\beta}\right)+\iota\left(\theta e_{\alpha}\right)_{i-1} \iota\left(\theta e_{\beta}\right)\right) \\
& +\frac{1}{4}\left(\iota\left(e_{\alpha}\right)_{i-1} \iota\left(\theta e_{\beta}\right)+\iota\left(\theta e_{\alpha}\right)_{i-1} \iota\left(e_{\beta}\right)\right) .
\end{aligned}
$$

Let us suppose $\langle\alpha, \beta\rangle<0$. Then

$$
\iota\left(e_{\alpha}\right)_{i-1} \iota\left(\theta e_{\beta}\right)=\iota\left(\theta e_{\alpha}\right)_{i-1} \iota\left(e_{\beta}\right)=0
$$

for $i \geq 0$. Therefore

$$
\iota\left(e_{\alpha}\right) *_{\theta} \iota\left(e_{\beta}\right) \equiv \frac{1}{4} \sum_{i=0}^{\frac{1}{2}\langle\alpha, \alpha\rangle}\left(\begin{array}{c}
\frac{1}{2}\langle\alpha, \alpha\rangle \\
i
\end{array}\right)\left(\iota\left(e_{\alpha}\right)_{i-1} \iota\left(e_{\beta}\right)+\iota\left(\theta e_{\alpha}\right)_{i-1} \iota\left(\theta e_{\beta}\right)\right) \bmod O_{\theta}\left(V_{L}\right) .
$$

Using Lemmas 3.8 and 3.10 produce scalar $c_{\alpha, \beta} \in \mathbb{C}$ such that $\iota\left(e_{\alpha}\right) *_{\theta} \iota\left(e_{\beta}\right) \equiv$ $c_{\alpha, \beta} \iota\left(e_{\alpha+\beta}\right) \bmod O_{\theta}\left(V_{L}\right)$. Evaluating this on the top levels of modules $V_{L}^{T_{\chi}}$ as in Lemma 3.10, we see $c_{\alpha, \beta}=\epsilon(\alpha, \beta) 2^{2\langle\alpha, \beta\rangle}$. In particular, $c_{\alpha, \beta} \neq 0$. 
If $\langle\alpha, \beta\rangle>0$, a similar argument shows that $\iota\left(e_{\alpha}\right) *_{\theta} \iota\left(e_{\beta}\right) \equiv c_{\alpha, \beta} \iota\left(e_{-\alpha+\beta}\right)$ for some scalar $c_{\alpha, \beta} \in \mathbb{C}$. By Lemma 3.10, we see $\iota\left(e_{-2 \alpha}\right) *_{\theta} \iota\left(e_{\alpha+\beta}\right) \equiv 2^{-4\langle\alpha, \alpha\rangle} \iota\left(e_{\alpha+\beta}\right)$. On the other hand, since $\langle-2 \alpha, \alpha+\beta\rangle=-2\langle\alpha, \alpha\rangle-2\langle\alpha, \beta\rangle<0$, we have $\iota\left(e_{-2 \alpha}\right) *_{\theta} \iota\left(e_{\alpha+\beta}\right) \equiv$ $c_{-2 \alpha, \alpha+\beta} \iota\left(e_{-\alpha+\beta}\right)$ for some nonzero constant $c_{-2 \alpha, \alpha+\beta}$. Thus $\iota\left(e_{\alpha}\right) *_{\theta} \iota\left(e_{\beta}\right) \equiv d_{\alpha, \beta} \iota\left(e_{\alpha+\beta}\right)$ with some scalar $d_{\alpha, \beta} \in \mathbb{C}$. Again as before we have $d_{\alpha, \beta}=\epsilon(\alpha, \beta) 2^{2\langle\alpha, \beta\rangle}$.

For $\alpha \in L$ set $u_{\alpha}=2^{\langle\alpha, \alpha\rangle} \iota\left(e_{\alpha}\right)$. Recall that $R$ is a sublattice of $L$.

Proposition 3.12. (1) For any $\alpha, \beta \in L$,

$$
u_{\alpha} * u_{\beta}=\epsilon(\alpha, \beta) u_{\alpha+\beta} \quad \bmod O_{\theta}\left(V_{L}\right) .
$$

(2) $u_{\alpha}+O_{\theta}\left(V_{L}\right)$ is in the center of $A_{\theta}\left(V_{L}\right)$ if and only if $\alpha \in R$.

Proof. (1) is what we have proved. For (2) we compare

$$
u_{\alpha} * u_{\beta}=\epsilon(\alpha, \beta) u_{\alpha+\beta} \quad \bmod O_{\theta}\left(V_{L}\right)
$$

and

$$
u_{\beta} * u_{\alpha}=\epsilon(\beta, \alpha) u_{\alpha+\beta} \quad \bmod O_{\theta}\left(V_{L}\right) .
$$

So $u_{\alpha}+O_{\theta}\left(V_{L}\right)$ is in the center if and only if $\epsilon(\alpha, \beta) \epsilon(\beta, \alpha)=(-1)^{\langle\alpha, \beta\rangle}=1$ or if and only if $\alpha \in R$.

Motivated by Proposition 3.12 we define a map $f: \mathbb{C}[\hat{L} / K] \rightarrow A_{\theta}\left(V_{L}\right)$ such that $f\left(e_{\alpha} K\right)=u_{\alpha}$ and $f((-1) K)=-1$. Then from Proposition 3.12, this is an onto algebra homomorphism and $(-1) K+1$ is in the kernel. Let $I$ be the two sided ideal of $\mathbb{C}[\hat{L} / K]$ generated by $(-1) K+1$. Then $f$ induces an onto algebra homomorphism $\bar{f}: \mathbb{C}[\hat{L} / K] / I \rightarrow A_{\theta}\left(V_{L}\right)$.

Our second main theorem in this paper is:

Theorem 3.13. (1) $A_{\theta}\left(V_{L}\right)$ is an finite-dimensional semisimple algebra isomorphic to $\mathbb{C}[\hat{L} / K] / I$.

(2) The irreducible $\theta$-twisted modules up to isomorphism are $V_{L}^{T_{\chi}}$ for all $T_{\chi}$ given in Proposition 3.4. Moreover, every irreducible admissible $\theta$-twisted $V_{L}$ is an ordinary $\theta$-twisted $V_{L}$ module.

(3) $V_{L}$ is $\theta$-rational. 
Proof. (1) First note from Proposition 3.4 and Theorem 3.5 that $\mathbb{C}[\hat{L} / K] / I$ is a finitedimensional semisimple algebra whose irreducible modules are precisely those $T_{\chi}$ which are the top levels of the irreducible $\theta$-twisted modules $V_{L}^{T_{\chi}}$ for $V_{L}$. So every irreducible module for $\mathbb{C}[\hat{L} / K] / I$ is an irreducible module for $A_{\theta}\left(V_{L}\right)$ and $\bar{f}$ must be an isomorphism.

(2) follows from Theorem 3.2 and part (1).

(3) By Theorem 3.3 it is enough to show that $L(0)$ has the same eigenvalue on all

$T_{\chi}$. In fact it is shown in $[\mathrm{FLM}]$ that $L(0)$ acts on all $T_{\chi}$ as $\frac{1}{16} d$ where $d$ is the rank of $L$.

\section{References}

[B] R. E. Borcherds, Vertex algebras, Kac-Moody algebras, and the Monster, Proc. Natl. Acad. Sci. USA 83 (1986), 3068-3071.

[D1] C. Dong, Vertex algebras associated with even lattices, J. Algebra 161 (1993), 245-265.

[D2] C. Dong, Twisted modules for vertex algebras associated with even lattice, J. Algebra 165 (1994), 91-112.

[DG] C. Dong and R.L. Griess Jr., Rank one lattice type vertex operator algebras and their automorphism groups, J. Algebra, to appear, q-alg/9710017.

[DGR] C. Dong, R.L. Griess Jr. and A. Ryba, Rank one lattice type vertex operator algebras and their automorphism groups, II E-series, preprint.

[DL1] C. Dong and J. Lepowsky, Generalized Vertex Algebras and Relative Vertex Operators, Progress in Math., Vol. 112, Birkhaüser, Boston, 1993.

[DL2] C. Dong and J. Lepowsky, The algebraic structure of relative twisted vertex operators, J. Pure and Applied Algebra 110 (1996), 259-295.

[DLM1] C. Dong, H. Li and G. Mason, Regularity of rational vertex operator algebras, Advances. in Math. 132 (1997), 148-166.

[DLM2] C. Dong, H. Li and G. Mason, Certain associative algebras similar to $U\left(s l_{2}\right)$ and Zhu's algebra $A\left(V_{L}\right)$, J. Algebra 196 (1997), 532-551. 
[DLM3] C. Dong, H. Li and G. Mason, Twisted representations of vertex operator algebras, Math. Ann. 310 (1998), 571-600.

[FHL] I. Frenkel, Y. Huang and J. Lepowsky, On axiomatic approaches to vertex operator algebras and modules, Memoirs Amer. Math. Soc. 104, 1993.

[FLM] I. Frenkel, J. Lepowsky and A. Meurman, Vertex Operator Algebras and the Monster, Pure and Appl. Math., Vol. 134, Academic Press, Boston, 1988.

[FZ] I.B. Frenkel and Y. Zhu, Vertex operator algebras associated to representations of affine and Virasoro algebras, Duke Math. J. 66 (1992), 123-168.

[Le] J. Lepowsky, Calculus of twisted vertex operators, Proc. Natl. Acad Sci. USA 82 (1985), 8295-8299.

[Li] H. Li, Local systems of vertex operators, vertex superalgebras and modules, $J$. Pure Appl. Alg. 109 (1996) 143-195.

[LX] H. Li and X. Xiao, A characterization of vertex algebras associated to even lattices, J. Algebra 173 (1995), 253-270.

[MN] A. Matsuo and K. Nagatomo, A note on free bosonic vertex algebra and its conformal vectors, J. Algebra, to appear, hep-th/9704060.

[W] W. Wang, Rationality of Virasoro vertex operator algebras, Duke Math. J. IMRN, Vol. 71, No. 1 (1993), 197-211.

[Z] Y. Zhu, Modular invariance of characters of vertex operator algebras, J. Amer, Math. Soc. 9 (1996), 237-302. 